Check for updates

Cite this: RSC Adv., 2017, 7, 43772

Received 24th July 2017

Accepted 24th August 2017

DOI: $10.1039 / \mathrm{c} 7 \mathrm{ra} 08148 \mathrm{k}$

rsc.li/rsc-advances

\section{Enhanced sludge anaerobic fermentation using microwave pretreatment combined with biosurfactant alkyl polyglycoside}

\begin{abstract}
Jianwei Xiao, ${ }^{\text {ab }}$ Liangjun Zhao (D)*b and Zhe Shen ${ }^{\mathrm{ab}}$
This study investigated the effect of microwave combined with biosurfactant alkyl polyglucose (APG) on sludge anaerobic fermentation in a lab-scale sequencing batch reactor. The experimental results showed that microwave combined with APG has a synergistic effect on sludge solubilization and methanogenesis, and that the optimal dosage of APG was $0.32 \mathrm{~g} \mathrm{~g}^{-1}$ TSS, the SCFA yield was $351.2 \mathrm{mg}$ COD/volatile suspended solids (VSS), which was 4.7-fold of that in blank. In addition, it was found that microwave pretreatment combined with APG can promote the activity of key enzymes, which was beneficial for the accumulation of SCFA. Microbial community analysis showed that the proportion of Firmicutes greatly improved due to the combination of microwave pretreatment and APG, and the percentage of Firmicutes in the microwave pretreatment \& APG reactor was 46.5\%, which was 3.1- and 1.1-fold of that in microwave pretreatment and APG reactor alone, respectively.
\end{abstract}

\section{Introduction}

The activated sludge process is the most widely used in wastewater treatment plants (WWTPs) because it has the characteristics of low operating cost, simple operation and high processing efficiencies. ${ }^{1,2}$ A large amount of waste activated sludge (WAS) is produced worldwide. It was reported that the amount of WAS in China is expected to rise to 34 million metric tonnes (at a moisture content of $80 \%$ ) in 2018 , and approximately $80 \%$ of it would be disposed unsafely, which would pose a great threat to the environment., ${ }^{3,4}$ Therefore, an effective sludge treatment method is urgently needed.

In recent years, sludge anaerobic digestion technology has been widely used and developed, sludge anaerobic digestion can reduce sludge volume, kill the pathogens in sludge and recover valuable resources such as short-chain fatty acid (SCFA), methane and hydrogen, simultaneously. ${ }^{5,6}$ SCFA is a preferred carbon source that can be used for biological denitrification and phosphorus removal and polyhydroxyalkanoate (PHA) synthesis. ${ }^{6}$ Sludge anaerobic fermentation generally involves four continuous steps: solubilization, hydrolysis, acidification and methanogenesis. ${ }^{7,8}$ The hydrolysis process is considered to be the rate-limiting step for sludge anaerobic fermentation because the extracellular polymer and the cell wall/membrane encapsulated outside the sludge limit the diffusion and release of intracellular organic matter. ${ }^{9,10}$ In addition, the SCFA

${ }^{a}$ Xinjiang Tianyu Beidou Satellite Technology Co., Ltd., Xinjiang Satellite Application Center, Urumqi, Xinjiang, 830000, P. R. China

${ }^{b}$ School of Resource and Environmental Science, Xinjiang University, Urumqi, Xinjiang, 830046, P. R. China. E-mail:27702792@qq.com produced by anaerobic fermentation could be rapidly consumed by methanogens. Therefore, increasing the rate of hydrolysis but inhibiting the activities of methanogens can improve the yield of SCFA. ${ }^{11,12}$ Thus, various sludge pretreatment technologies such as thermal, chemical, mechanical, ultrasonic, enzymatic methods and their combinations have been proposed to improve the production of intermediates. ${ }^{13,14}$

Biosurfactants have the characteristics of fast biodegradability and good ecological compatibility. Alkyl polyglycoside (APG) is a mild non-ionic surfactant, but it has the properties of both non-ionic and anionic surfactants. APG can be derived from renewable plants such as corn, coconut oil, and palm kernel oil. In addition, APG does not contain $\mathrm{K}^{+}, \mathrm{Na}^{+}$and other metal ions, and hence it can be completely degraded to $\mathrm{H}_{2} \mathrm{O}$ and $\mathrm{CO}_{2}$. As an environmentally friendly surfactant, APG can promote the processes of solubilization and hydrolysis during organic matter anaerobic digestion, thereby reducing the time of the anaerobic digestion process. Zhao et al. (2015) investigated the effect of APG on the treatment of membrane sludge anaerobic fermentation, and it was found that when the dosage of APG was $0.2 \mathrm{~g} \mathrm{~g}^{-1}$ dry sludge, the yield of SCFA was $282.9 \mathrm{mg}$ COD/g VSS, which was much higher than that in blank. ${ }^{13}$ In addition, they noted that the efficiency in alkaline environments with APG was higher than that in neutral or acidic environments, and the optimum $\mathrm{pH}$ was 11 . Similar experimental results were also obtained by Luo et al. (2015). ${ }^{15}$ In view of the unique properties of APG, using APG to treat sludge is a feasible and promising strategy. Although biosurfactant APG is capable of dissolving sludge, the degree of sludge disintegration is unsatisfactory, which limits the further use of organic matter in the sludge. 
Recently, microwave (MW) pretreatment has served as an effective and environmentally friendly technology and is widely used in the disposal of organic matter. MW technology is capable of disrupting sludge flocs and cells and releasing organic matter into the soluble phase. ${ }^{\mathbf{1 6 , 1 7}}$ Unlike traditional heating methods, microwave heating is from the inside to the outside of the material, thus reducing energy loss. ${ }^{16}$ Appels et al. investigated the effect of microwave pretreatment on sludge dissolution, and they found that microwave pretreatment could promote sludge dissolution and lead to a $50 \%$ increase in biogas production..$^{18}$ It was also found that the ratio of SCOD to TCOD increased from $2 \%$ to $22 \%$ from sludge anaerobic digestion after MW pretreatment (2450 MHz, 700 W for $15 \mathrm{~min}$ ), and the biochemical acidogenic potentials also increased from 3.58 to $4.77 \mathrm{~g} \mathrm{COD} / \mathrm{L} .{ }^{19}$ Microwave pretreatment together with other treatment techniques such as alkaline, hydrogen peroxide, etc. have also been reported to promote sludge solubilization and biogas production. ${ }^{20,21}$ The nature of biosurfactants is unlike chemical reagents such as sodium hydroxide. Whether or not microwave pretreatment combined with biosurfactants can promote the solubilization of sludge and the accumulation of SCFA has not been reported, and its impact on microbial communities is also unclear.

Therefore, the aim of this study is to investigate the effect of MW combined with biosurfactant APG on sludge anaerobic fermentation. First, SCFA production was investigated in the presence of varying APG dosage. Then, the mechanism of microwave combined with APG on sludge anaerobic fermentation was explored from the aspects of sludge solubilization, acidification, methanogenesis, and activities of the key enzyme. Finally, the effect of MW combined with APG on the changes of microbial community structure in the sludge fermentation system was investigated. The findings achieved in this study might help to recover bio-energy from WAS anaerobic fermentation and have significant implications for the real operation of WAS.

\section{Materials and methods}

\subsection{WAS source, inoculum, APG and MW}

WAS was obtained from the secondary sedimentation tank in Urumqi and stored in the laboratory for $24 \mathrm{~h}$. The supernatant was removed and stored in a refrigerator at $4{ }^{\circ} \mathrm{C}$ for further use. The main characteristics (average value plus standard deviation of three tests) of WAS are presented as follows: pH $6.9 \pm 0.1$, total suspended solids (TSS) $15110 \pm 160 \mathrm{mg} \mathrm{L}{ }^{-1}$, volatile suspended solids (VSS) $8900 \pm 210 \mathrm{mg} \mathrm{L}^{-1}$, soluble COD $150 \pm$ $16 \mathrm{mg} \mathrm{L}^{-1}$, total protein $6652 \pm 280 \mathrm{mg} \mathrm{L}^{-1}$, and total carbohydrate $930 \pm 200 \mathrm{mg} \mathrm{L}^{-1}$.

The inoculum was taken from an upflow anaerobic sludge blanket in our laboratory; the main characteristics of the inoculum were as follows: pH $6.8 \pm 0.1$, total suspended solids (TSS) $16520 \pm 160 \mathrm{mg} \mathrm{L}^{-1}$, and volatile suspended solids (VSS) 10900 $\pm 210 \mathrm{mg} \mathrm{L}^{-1}$.

APG used in this study was purchased from Shanghai Chemical Pharmaceutical Co., Ltd.; the main characteristics of APG were as follows: solid content $50 \%$ and density $1.10 \mathrm{~g} \mathrm{~cm}^{-3}$.
The microwave equipment used in the test was a selfdeveloped microwave reactor with a frequency of $2500 \mathrm{MHz}$ and magnetron maximum output power and temperature of $1 \mathrm{~kW}$ and $100{ }^{\circ} \mathrm{C}$, respectively. The reaction vessel is a plastic container with a volume of $2.0 \mathrm{~L}$ and the upper part of the container is open, which is equipped with a stirrer for homogenization and a thermocouple temperature sensor.

\subsection{Comparison of SCFA production from WAS anaerobic fermentation by APG and MW}

The dosage of APG is critical to SCFA production from sludge fermentation. Therefore, this study first explored the effect of APG dosage on SCFA production from sludge anaerobic fermentation with MW pretreatment. Herein, 4.0 L of WAS was first heated to $100{ }^{\circ} \mathrm{C}$ with $600 \mathrm{~W}$ of microwave power at atmospheric pressure. The microwave pretreatment frequency selection at 600 is based on a previous study, which reported that the disintegration of sludge was effective when the frequency of the MW was at $600 \mathrm{~W} .^{22,23}$ The WAS was divided in five identical reactors with a working volume of $1.0 \mathrm{~L}$ each, and then $0.2 \mathrm{~L}$ of the inoculum was inoculated into each reactor. The ratio of the inoculum to substrate was based on a previously reported value in a study with minor change. ${ }^{2}$ Finally, different dosages of APG were added to the reactors to control the APG content of $0,0.02,0.08,0.32$ and $0.64 \mathrm{~g} \mathrm{~g}^{-1} \mathrm{TSS}$, respectively. The initial organic load in each reactor remained the same by discharging the sludge properly. In addition, in order to investigate the contribution of APG degradation to SCFA production, another reactor without the digestive matrix was employed. The reactor was first inoculated with $0.2 \mathrm{~L}$ of inoculated sludge; then, $0.8 \mathrm{~L}$ of distilled water was manually added, followed by a certain amount of APG with the concentration being controlled at $0.32 \mathrm{~g} \mathrm{~g}^{-1}$ TSS. Other experimental conditions are described above. These reactors were then purged with nitrogen for $60 \mathrm{~s}$ to remove oxygen, sealed and placed on an air shaker (120 rpm) for $30 \mathrm{~d}$.

\subsection{Mechanism of MW pretreatment combined with APG enhanced SCFA production}

In order to investigate the mechanism of microwave combined with APG for sludge solubilization and SCFA production. Three identical reactors were employed; WAS in two reactors was treated with APG or MW pretreatment, separately. In the MW pretreatment reactor, WAS was first heated to $100^{\circ} \mathrm{C}$ with $600 \mathrm{~W}$ microwave power at atmospheric pressure and then fermented at $35^{\circ} \mathrm{C}$. In the APG reactor, the dosage of APG was controlled at $0.32 \mathrm{~g} \mathrm{~g}^{-1}$ TSS. The WAS in another reactor was MW pretreated combined APG. Other operational conditions are described in Section 2.2.

\subsection{Effects of MW pretreatment combined with APG on methanogenesis}

This batch experiment was used to investigate the effect of microwave treatment combined with APG on the process of methanogenesis. Three identical reactors were employed. Each of the three reactors first received $200 \mathrm{~mL}$ of inoculum and 
$800 \mathrm{~mL}$ of synthetic wastewater. The inoculum of one reactor (defined as MW reactor) was subjected to MW pretreatment; the detailed information of MW pretreatment is given in Section 2.3. One reactor (defined as APG reactor) was added with APG, and the inoculum of another reactor (defined as microwave \& APG reactor) was first subjected to microwave pretreatment and then added with APG. The detailed information of synthetic wastewater is as follows: NaAc $\left(1.0 \mathrm{~g} \mathrm{~L}^{-1}\right)$, potassium phosphate (50 mM, pH 7.0), $\mathrm{KCl}\left(0.13 \mathrm{~g} \mathrm{~L}^{-1}\right), \mathrm{NH}_{4} \mathrm{Cl}\left(0.31 \mathrm{~g} \mathrm{~L}^{-1}\right)$, Wolf's trace element solution $\left(0.5 \mathrm{~mL} \mathrm{~L}^{-1}\right)$ and vitamin solution $\left(0.5 \mathrm{~mL} \mathrm{~L}^{-1}\right)$.

\subsection{Long-term operation of the semi-continuous reactor to} detect the key enzymes activities and microbial communities

In this experiment, three identical semi-continuous flow reactors were employed with a working volume of $1.0 \mathrm{~L}$ per reactor. The basic operating conditions of the semi-continuous flow reactor were the same as those of the SBR except for the following description. According to the experimental results obtained (Section 2.2), the maximum yield of SCFA was obtained on $5 \mathrm{~d}$, and thus the sludge residence time of the semicontinuous flow was controlled at $5 \mathrm{~d}$. Furthermore, $200 \mathrm{~mL}$ of sludge was manually removed from the reactor and the same volume of fresh sludge was added daily. The sludge in one reactor was subjected to microwave pretreatment only, and another reactor was charged with APG only. The sludge in the last reactor was subjected to microwave pretreatment combined with APG. It took approximately three months to reach the stable SCFA production, and then the sludge was subjected to key enzyme activity determination and microbial community identification.

\subsection{Analytical methods}

The detections of TSS, VSS, COD, SCOD, and pH were according to the standard method. ${ }^{24}$ Protein and polysaccharides were measured using the Lowry-Folin method with BSA and glucose as the standard substrates, respectively. ${ }^{25,26}$ Gas chromatography was used to detect the content and composition of SCFA, and the gas chromatographic model was Agilent 6890-DBMAXETR. Determination of the activity of key enzymes responsible for SCFA production has been shown in a literature reported previously. ${ }^{27}$ The determination procedure of coenzyme F420 was similar to that reported in the literature. ${ }^{28}$ DNA was extracted from the biomass of each anaerobic reactor using the E.Z.N.A Soil DNA Kit (Omega Bio-Tek. Inc., Georgia, USA). Samples were centrifuged at $10000 \mathrm{rpm}$ for $5 \mathrm{~min}$ before extraction. Each extraction was performed in duplicate, and the extracted DNA was eluted in Tris-HCl buffer ( $\mathrm{pH}$ 8.0) and stored at $20{ }^{\circ} \mathrm{C}$. Detailed extraction procedures can be obtained in the literature. $^{29-32}$ The Illumina Miseq sequencing technique was applied to identify microbial communities, and the detailed information was recorded in literature..$^{33,34}$

\subsection{Statistical analysis}

All experiments were repeated three times. Analysis of variance was employed to evaluate the significance of the results, and $p<$ 0.05 was considered to be statistically significant.

\section{Results and discussion}

\subsection{Effect of APG dosage on the yield of SCFA from WAS anaerobic fermentation}

The addition of APG was found to critical to the production of SCFA from sludge anaerobic fermentation. Fig. 1 shows the effect of APG dosage on the yield of SCFA with fermentation time in the presence of MW pretreatment. As can be seen from Fig. 1, the production of SCFA in each reactor showed a tendency to first rise and then decline with the time. In the blank, the maximum value of SCFA was $73.9 \mathrm{mg}$ COD/g VSS. However, when the concentration of APG increased from 0.02 to $0.32 \mathrm{~g} \mathrm{~g}^{-1} \mathrm{TSS}$, the maximum value of SCFA increased from $142.5 \mathrm{mg} \mathrm{L}^{-1}$ to $351.2 \mathrm{mg}$ COD/g VSS; the corresponding SCFA maximum content also increased from 1.9 to 4.7 -fold of that in blank. Further increase of APG dosage, however, did not significantly increase the SCFA content $(p>0.05)$. The accumulation of SCFA is closely related to hydrolysis, acidification and methanogenesis. Because APG poses a serious inhibitory effect on methane production, and further increase of APG dosage does not enhance the degree of inhibition. ${ }^{13}$ In addition, Luo et al. (2015) found that the effect of APG on hydrolysis and acidification was obvious when the APG dosage exceeded $0.3 \mathrm{~g} \mathrm{~g}^{-1} \mathrm{TSS}^{15}$ Furthermore, the contribution of APG degradation to SCFA production was negligible (see Section 3.3). Therefore, the accumulations of SCFA were similar at 0.32 and $0.64 \mathrm{~g} \mathrm{~g}^{-1}$ TSS APG in this study. The fermentation time has significant implications for practical engineering, and shortening the fermentation time means that the volume of the fermentation reactor can be reduced and the residence time of the solid can be shortened. From Fig. 1, it can be observed that the maximum accumulation time of SCFA in the blank was obtained on $7 \mathrm{~d}$, and when the APG was added into the WAS fermentation system, the optimum fermentation time was shortened to $5 \mathrm{~d}$. Luo et $a .^{15}$ reported that the optimal time for

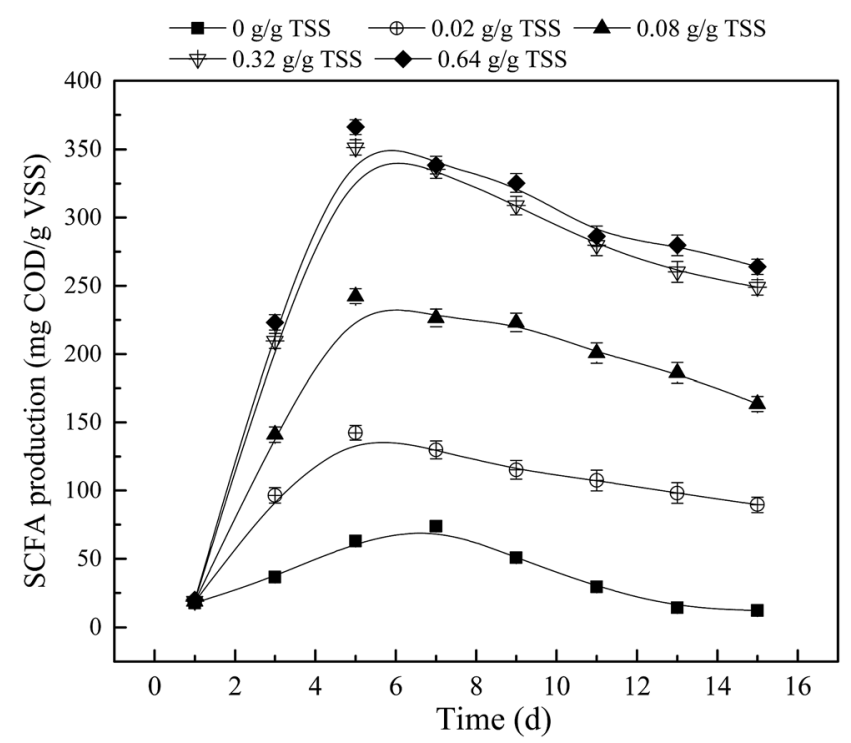

Fig. 1 Effect of APG dosage on the yield of SCFA from WAS anaerobic fermentation. Error bars represent standard deviation. 
APG to produce propionic acid was $4 \mathrm{~d}$. The main reason for the difference may be that the organic matter content in the sludge in this study was low. Low organic matter content can cause a decrease of SCFA production. ${ }^{28}$ The above results clearly showed that APG can promote the accumulation of SCFA and shorten the optimal fermentation time.

\subsection{Effect of APG dosage on the SCFA composition}

The composition of SCFA plays a vital role in its subsequent utilization. It is generally believed that acetic acid is the preferred carbon source for the biological denitrification process, and propionic acid is the preferred carbon source for phosphorusremoval microorganisms. ${ }^{35}$ Fig. 2 shows the effect of APG dosage on the composition of SCFA from sludge anaerobic fermentation. Acetic acid and propionic acid were the main components, the sum percentage of acetic acid and propionic acid was about $82-91 \%$ in all reactors, and the content of isovaleric acid was the lowest. It should be noted that when the dosage of APG was low, acetic acid was the main component, and when the dosage of APG exceeded $0.08 \mathrm{~g} \mathrm{~g}^{-1}$ TSS, propionic acid became the predominant first component. The above analysis shows that the appropriate increase in the dosage of APG would be beneficial to improve the propionic acid percentage. It is reported that the content of carbon in APG is high and the content of $\mathrm{N}$ in the sludge is high. When APG was added into the sludge system, the $\mathrm{C} / \mathrm{N}$ of the digestive matrix was improved, which was beneficial to the microbial activity and the production of propionate. The increase in propionic acid percentage helps the SCFA to be used for biological phosphorus removal.

\subsection{The contribution of APG degradation to SCFA}

The biosurfactant APG can be degraded during anaerobic fermentation. In this study, SCFA may be derived from two

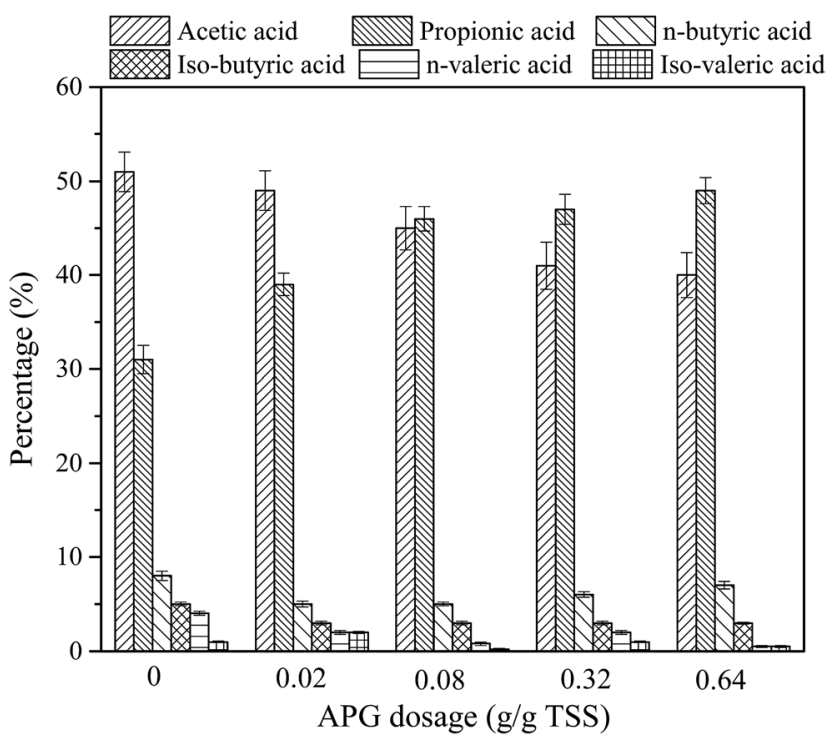

Fig. 2 Effect of APG dosage on SCFA components. The detection time is when the SCFA reaches the maximum value in each reactor. Error bars represent standard deviation. aspects: WAS fermentation and degradation of APG. Therefore, it is necessary to explore the contribution of APG degradation to SCFA production. Fig. 3 shows the amount of SCFA produced from the degradation of individual WAS and APG alone. It can be seen from Fig. 3 that the maximum amount of SCFA produced by sludge and APG alone is 658 and $415 \mathrm{mg} \mathrm{L}^{-1}$, respectively. However, when WAS + APG was fermented, the highest production of SCFA reached $3126 \mathrm{mg} \mathrm{L}^{-1}$, which was significantly higher than the sum of that from WAS and the APG fermentation alone. The above results indicate that the contribution of SCFA from APG degradation is small, and thus APG has a positive effect on the production of SCFA from WAS anaerobic fermentation.

\subsection{Effect of microwave combined with APG on WAS solubilization}

The organic matter in WAS is usually in the form of granules or macromolecules, and the organic substances are first dissolved before being used. ${ }^{36,37}$ Fig. 4 shows the effect of microwave combined APG on WAS solubilization. During the first $5 \mathrm{~d}$, the content of SCOD in each reactor showed an increasing trend, indicating that both microwave and APG could promote the solubilization of WAS. When the MW and APG were present alone, the maximum content of SCOD was 1254 and $569 \mathrm{mg} \mathrm{L}^{-1}$, respectively. However, when the MW and APG were combined, the maximum value of SCOD increased to $3589 \mathrm{mg} \mathrm{L}^{-1}$. The above results clearly showed that the combination of MW and APG could produce a synergistic effect on WAS solubilization. Both APG and MW pretreatment can enhance the solubilization process of sludge, but their mechanisms of promoting solubilization are quite different. APG is a biosurfactant, and it can increase the solubility of organic matter by reducing the surface tension of hydrophobic molecules and water as it has hydrophobic and hydrophilic amphiphilic molecules. MW treatment mainly increases the temperature to the boiling point by increasing the dipole kinetic energy of the water molecule. Although the energy of the microwave is not

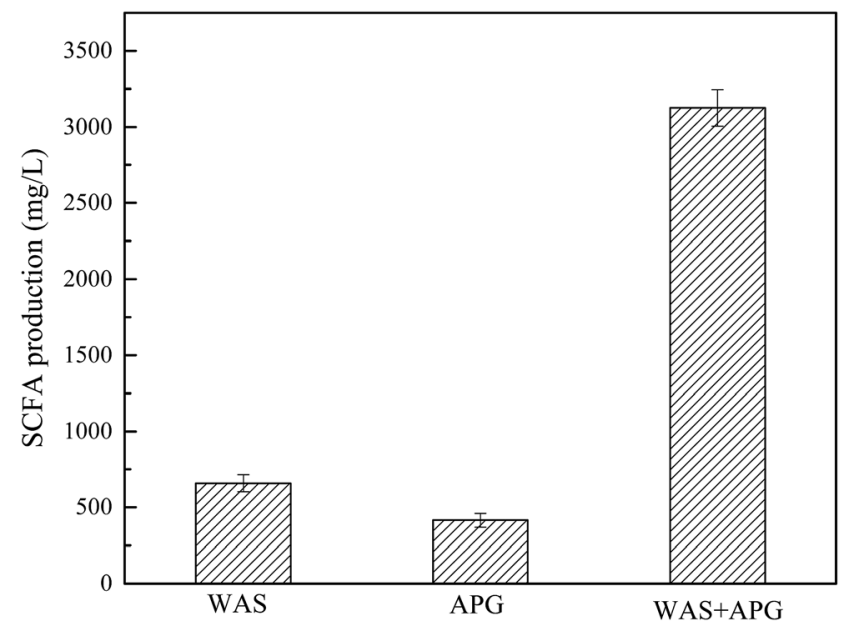

Fig. 3 Comparison of SCFA production from WAS, APG and WAS + $A P G$ anaerobic fermentation. Error bars represent standard deviation. 


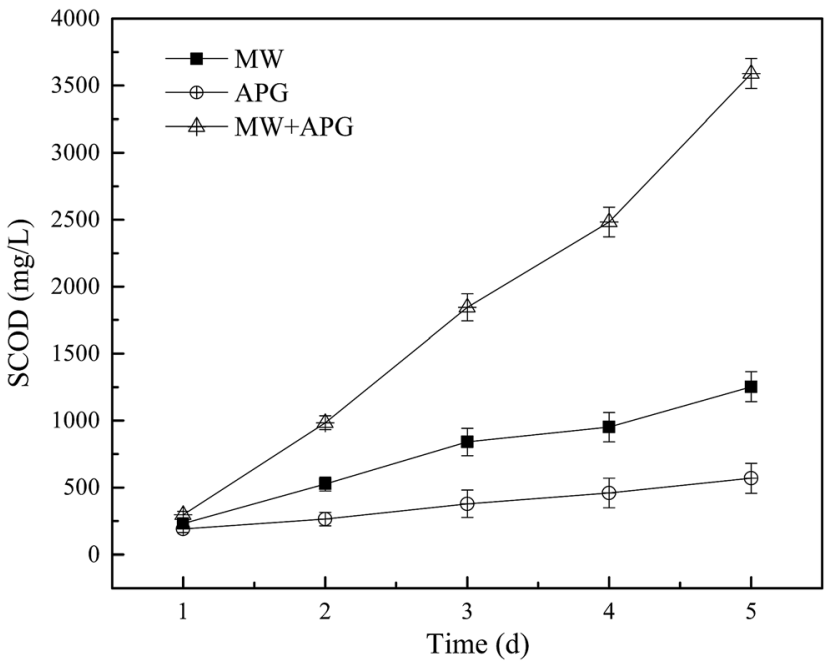

Fig. 4 Effect of MW combined with APG on the variations of SCOD during WAS anaerobic digestion. Error bars represent standard deviation.

sufficient to open the chemical bond, the structure of some hydrogen bonds can be weakened or even destroyed. The combination of MW and APG can promote the solubilization of organic matter in the sludge, thus improving the disintegration process of sludge.

Soluble protein and polysaccharide content changes can also reflect the sludge solubilization process. Table 1 shows the changes of soluble protein and polysaccharide with time in different reactors. As can be seen from Table 1, the concentrations of soluble protein and polysaccharide in each reactor showed a tendency of first rise and then decrease. When the MW pretreatment was applied alone, the maximum contents of soluble protein and polysaccharide were 425 and $185 \mathrm{mg} \mathrm{L}^{-1}$, respectively, and the optimum time was $3 \mathrm{~d}$. When the APG was present alone, the maximum contents of the soluble protein and polysaccharide were 335 and $174 \mathrm{mg} \mathrm{L}^{-1}$ on $3 \mathrm{~d}$, respectively. However, when the MW and APG were combined, the maximum values of soluble protein and polysaccharide increased to 651 and $254 \mathrm{mg} \mathrm{L} \mathrm{L}^{-1}$, which were significantly higher than those of MW or APG alone. In addition, the time when the soluble protein and polysaccharide content reached the maximum value was $2 \mathrm{~d}$, which was shorter than that of MW or APG alone. The above experimental results show that the combination of MW and APG has a synergistic effect on the solubilization of protein and polysaccharide.

\subsection{Effect of MW combined with APG on methanogenesis}

Methanogenesis is the last step involved in the anaerobic digestion of WAS, during which methanogens consume acidification products such as acetic acid and hydrogen to produce methane. $^{38,39}$ The production of methane is related to the accumulation of SCFA. Fig. 5 displays the effect of MW combined with APG on the degradation of acetic acid in synthetic wastewater. It can be seen from Fig. 5 that the content of acetic acid in each reactor tended to decrease with time, which was found to be mainly consumed for the production of methane by methanogens and the maintenance of metabolic activities. When the MW was applied alone, sodium acetate was rapidly consumed, and on $5 \mathrm{~d}$, the sodium acetate concentration was only $0.05 \mathrm{~g} \mathrm{~L}^{-1}$, indicating that the utilization rate of sodium acetate was as high as $95 \%$. When APG was used alone, the consumption of sodium acetate was severely inhibited, and on $5 \mathrm{~d}$, the concentration of sodium acetate was $0.64 \mathrm{~g} \mathrm{~L}^{-1}$. Zhao et al. $(2015)^{35}$ have reported that the presence of APG had an inhibitory effect on methanogens during food waste anaerobic fermentation. It was reported that the surfactants inhibited the

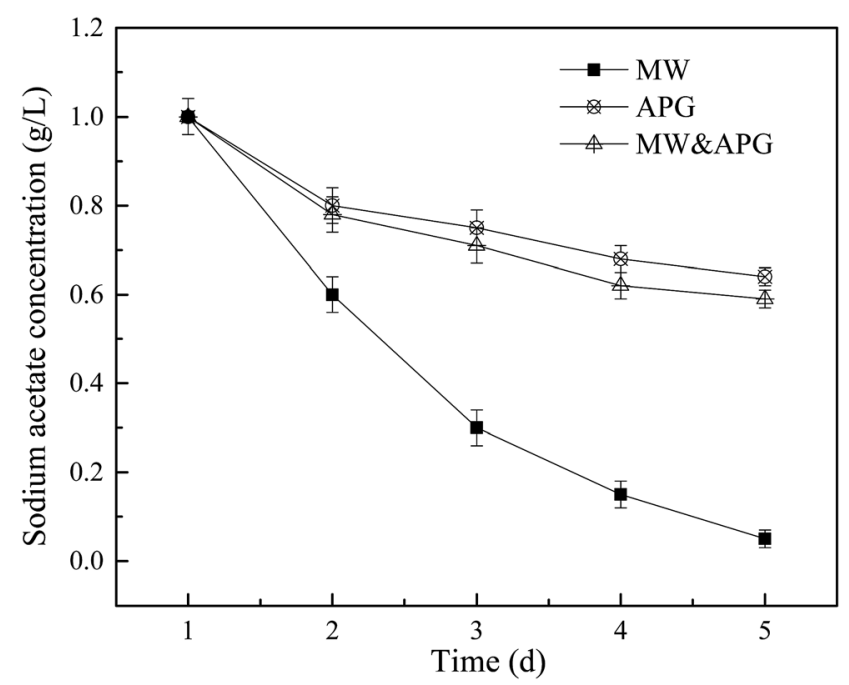

Fig. 5 Effect of MW combined with APG on the degradation of sodium acetate in synthetic wastewater. Error bars represent standard deviation.

Table 1 Effect of microwave combined with APG on solubilized proteins and polysaccharides over time ${ }^{a}$

\begin{tabular}{|c|c|c|c|c|c|c|}
\hline Treatment & & $1 \mathrm{~d}$ & $2 \mathrm{~d}$ & $3 \mathrm{~d}$ & $4 \mathrm{~d}$ & $5 \mathrm{~d}$ \\
\hline \multirow[t]{2}{*}{ MW } & Soluble protein & $117 \pm 6$ & $259 \pm 8$ & $425 \pm 19$ & $315 \pm 16$ & $245 \pm 14$ \\
\hline & Soluble polysaccharide & $65 \pm 3$ & $142 \pm 7$ & $185 \pm 9$ & $161 \pm 8$ & $145 \pm 8$ \\
\hline & Soluble polysaccharide & $59 \pm 2$ & $115 \pm 6$ & $174 \pm 9$ & $152 \pm 7$ & $112 \pm 6$ \\
\hline \multirow[t]{2}{*}{ MW\&APG } & Soluble protein & $117 \pm 6$ & $651 \pm 35$ & $452 \pm 24$ & $316 \pm 16$ & $236 \pm 12$ \\
\hline & Soluble polysaccharide & $63 \pm 3$ & $245 \pm 14$ & $174 \pm 10$ & $152 \pm 7$ & $102 \pm 6$ \\
\hline
\end{tabular}

${ }^{a}$ Error bars represent standard deviation. 


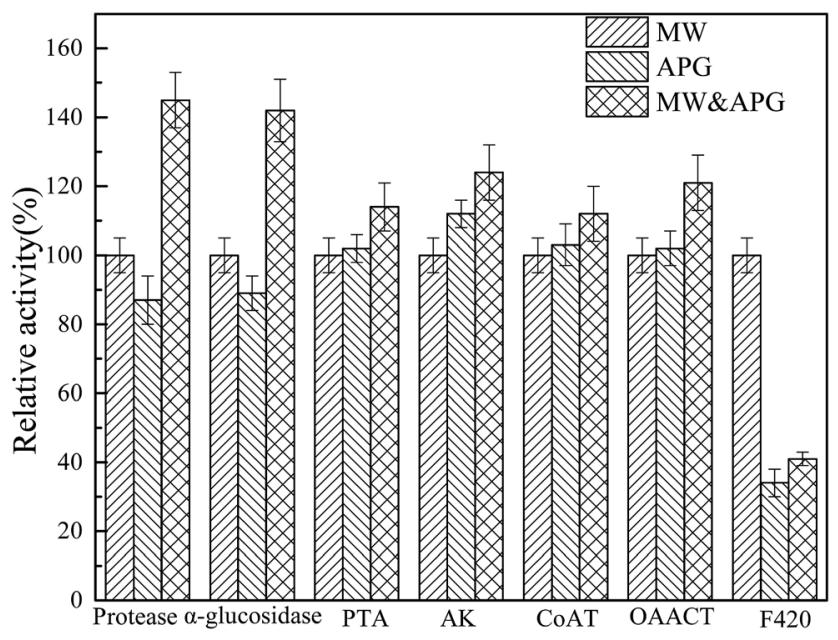

Fig. 6 Effects of microwave combined with APG on the activities of key enzymes responsible for SCFA production. The activities of the related enzymes in the microwave treatment group was $100 \%$. Error bars represent standard deviation.

activities of methanogens in the anaerobic system by disrupting their cell membranes. ${ }^{40}$ When microwave and APG were combined, the concentration of sodium acetate was $0.59 \mathrm{~g} \mathrm{~L}^{-1}$ on $5 \mathrm{~d}$, and the consumption of sodium acetate was still seriously inhibited. This result indicated that the effect of microwave introduction on the consumption of sodium acetate in the APG-induced system was not obvious.

\subsection{Effect of MW combined with APG on the activities of key enzymes and microbial communities}

The production of SCFA from WAS anaerobic fermentation is a biological process, which is mainly regulated by key enzymes and microbes. Protease and $\alpha$-glucosidase are the main key enzymes related to the hydrolysis of protein and carbohydrate, respectively. ${ }^{25}$ The activities of phosphotransferase (PTA), acetate kinase (AK), CoA transferase (CoAT), and oxaloacetate trans carboxylase (OAACT) are responsible for acetic acid and propionic acid production. F420 is a coenzyme unique to methanogens, and hence the activity of F420 can be used to indicate the activity of methanogens. Fig. 6 displayed the effect of MW combined APG on the activities of key enzymes involved in SCFA production. As shown in Fig. 6, the relative activities of key enzymes related to hydrolysis and acidase were significantly higher than those of microwave or APG alone. It is clearly understood why MW combined with APG can promote WAS hydrolysis and acidification. The activities of F420 in the microwave combined with the APG reactor were similar to that in the APG reactor alone, which was also one reason for SCFA accumulation in the MW combined with the APG reactor.

Microbial communities play an important role in the production of SCFA from WAS anaerobic fermentation. Therefore, this study also explored the effect of microwave combined with APG on microbial communities in WAS fermentation. As shown in Fig. 7, Proteobacteria, Bacteroidetes, Chloroflexi and Firmicutes were the main phyla in each reactor, and their percentages in MW, APG, and MW \& APG were 64.4\%, 85.9\%, and $93.6 \%$, respectively. These phyla have also been found in the conventional sludge fermentation system., ${ }^{2,41}$ Further investigation shows that the combination of MW with APG can increase the proportion of Firmicutes, and the percentage of Firmicutes in the MW + APG reactor was $46.5 \%$, which was 3.1 and 1.1-fold of that in the MW and APG reactor alone, respectively. It was well known that Firmicutes was able to excrete hydrolases to degrade a wide range of complex organic macromolecules, including both proteins and carbohydrates, and produce propionic and acetic acids through the propionic acid pathway. ${ }^{\mathbf{1 5 , 4 2}}$ The enrichment of Firmicutes was also a reason for the enhancement of WAS anaerobic fermentation with MW \& APG treatment. Proteobacteria was another key microorganism in WAS hydrolysis and SCFAs production, but it was also identified as the main consumer for acetate propionate and butyrate during WAS anaerobic fermentation. As shown in Fig. 7, the percentage of Proteobacteria in the MW reactor was $18.5 \%$; however, when MW \& APG was applied, the percentage of Proteobacteria decreased to $15.4 \%$, suggesting that the consumption of SCFA was reduced.

Fig. 8 further displays the effect of MW pretreatment combined with APG on microbial biomass at the genus-level distributions from sludge anaerobic fermentation. As shown in Fig. 8, the combination of microwave and APG improved the relative abundance of functional microorganisms such as Lactobacillus, Collinsella. When the MW and APG were applied
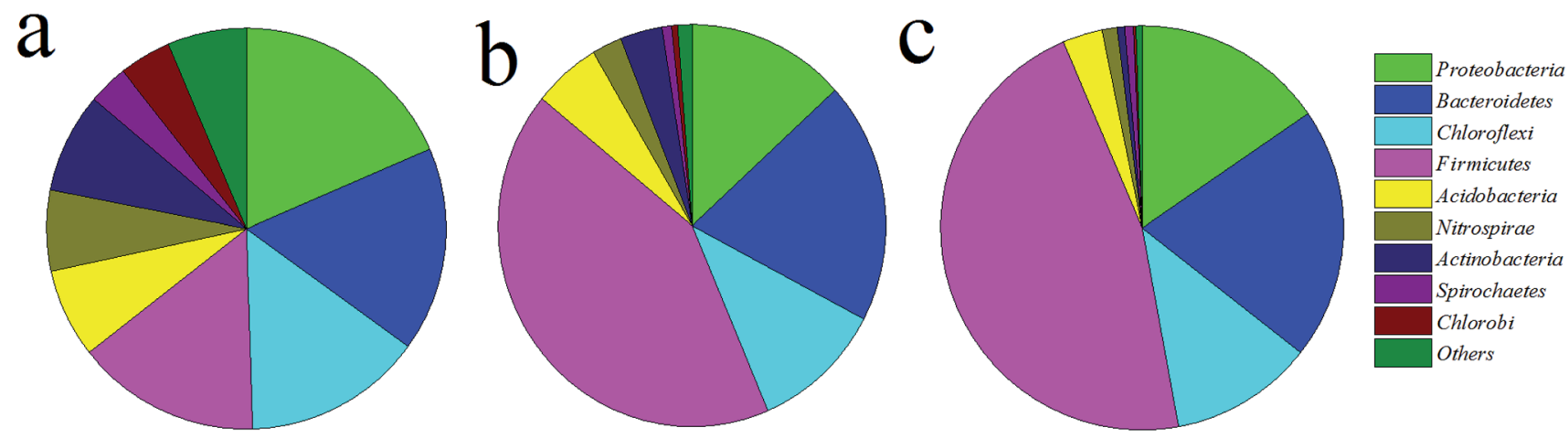

Fig. 7 Effects of microwave combined with APG on microbial communities at the phylum level ((a) MW; (b) APG; (c) MW + APG). 


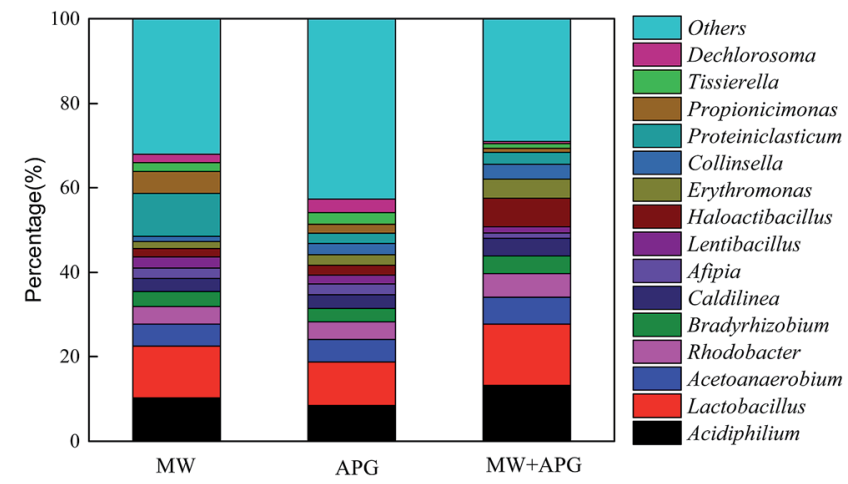

Fig. 8 Effects of microwave combined with APG on microbial communities at the genus-level.

alone, the relative abundance of Lactobacillus was $10.2 \%$ and $8.5 \%$. When the microwave and APG were combined, however, the relative abundance of Lactobacillus increased to $13.2 \%$. Similar experimental results are also obtained for Collinsella. Lactobacillus and Collinsella are important genera in the process of sludge anaerobic fermentation, and they are closely related to the decomposition of protein and the synthesis of SCFA. $^{3}$ It is clear that the combination of MW and APG contribute to the increase in the relative abundance of functional microorganisms during the anaerobic fermentation of sludge.

\subsection{Practical application of MW combined with APG to enhance sludge anaerobic fermentation}

With the acceleration of the urbanization process, the amount of waste activated sludge has increased and become a serious environmental problem. Anaerobic fermentation of sludge can result in high concentration of SCFA, which can be used as a carbon source for biological denitrification and denitrification system, improving the efficiency of biological nitrogen removal of low-carbon and high-nitrogen municipal wastewater. ${ }^{43-45}$ At the same time, anaerobic fermentation can achieve the reduction and harmless of the sludge.

APG is a mild nonionic surfactant, but it also has the characteristics of anionic surfactants. APG has many excellent properties, such as being foam-rich, exhibits low irritation, and is non-toxic, fully biodegradable, and can be easily accessed. The combination of MW and APG can produce a synergistic effect on sludge anaerobic fermentation, which promotes the hydrolysis of sludge and the accumulation of SCFA. When the APG dose was $0.32 \mathrm{~g} \mathrm{~g}^{-1}$ TSS, the optimal yield of SCFA was $351.2 \mathrm{mg}$ COD/g VSS, which is much higher than that reported with only APG..$^{15}$ The increase of SCFA production will bring huge economic benefits, despite MW consuming energy. In addition, APG would be completely degraded in an anaerobic environment resulting in $\mathrm{CO}_{2}$ and $\mathrm{H}_{2} \mathrm{O}$ as products, which will not pose a risk to environmental ecology and human health. Therefore, the combination of MW and APG for sludge treatment is a promising technology.

\section{Conclusion}

In this study, the effect of microwave pretreatment combined with APG on the production of SCFA in sludge anaerobic fermentation was systematically investigated. Experimental results showed that the combination of microwave and APG had a synergistic impact on sludge anaerobic fermentation, and the optimal dosage of APG was $0.32 \mathrm{~g} \mathrm{~g}^{-1}$ TSS; the SCFA yield was $351.2 \mathrm{mg}$ COD/g VSS, which was 4.7 -fold of that in blank. The combination of microwave pretreatment and APG had a synergistic impact on sludge solubilization and methanogenesis. Microbial community analysis showed that the proportion of Firmicutes greatly improved due to the combination of MW pretreatment and APG.

\section{Conflicts of interest}

There are no conflicts to declare.

\section{Acknowledgements}

This work was financially supported by Foundation item: Innovation Project of Doctoral Candidate of Xinjiang University, No. XJUBSCX-2014014.

\section{References}

1 A. Anbalagan, S. Schwede, C. F. Lindberg and E. Nehrenheim, Water Res., 2016, 91, 277-284.

2 J. Zhao, L. Gui, Q. Wang, Y. Liu, D. Wang, B. J. Ni, X. Li, R. Xu, G. Zeng and Q. Yang, Water Res., 2017, 123, 724-733.

3 Q. Xu, X. Li, R. Ding, D. Wang, Y. Liu, Q. Wang, J. Zhao, F. Chen, G. Zeng, Q. Yang and H. Li, Water Res., 2017, 124, 269-279.

4 J. Luo, Y. Chen and L. Feng, Environ. Sci. Technol., 2016, 50(13), 6921-6929.

5 J. Zhao, D. Wang, X. Li, Q. Yang, H. Chen, Y. Zhong and G. Zeng, Water Res., 2015, 78, 111-120.

6 D. Wang, Y. Chen, X. Zheng, X. Li and L. Feng, Environ. Sci. Technol., 2013, 47(6), 2688-2695.

7 Y. Yan, L. Feng, C. Zhang, C. Wisniewski and Q. Zhou, Water Res., 2010, 44(11), 3329-3336.

8 J. Zhao, C. Zhang, D. Wang, X. Li, H. An, T. Xie, F. Chen, Q. Xu, Y. Sun, G. Zeng and Q. Yang, ACS Sustainable Chem. Eng., 2016, 4(9), 4675-4684.

9 H. Carrère, C. Dumas, A. Battimelli, D. J. Batstone, J. P. Delgenès, J. P. Steyer and I. Ferrer, J. Hazard. Mater., 2010, 183(1), 1-15.

10 A. Lagerkvist and F. Morgan-Sagastume, Waste Manag, 2012, 32(9), 1634-1650.

11 Y. Chen, H. Liu, X. Zheng, X. Wang and J. Wu, Appl. Energy, 2017, 196, 190-198.

12 S. Kavitha, C. Jayashree, S. A. Kumar, I. T. Yeom and J. R. Banu, Bioresour. Technol., 2014, 168, 159-166.

13 J. Zhao, Q. Yang, X. Li, D. Wang, H. An, T. Xie, Q. Xu, Y. Deng and G. Zeng, Int. Biodeterior. Biodegrad., 2015, 104, 283-289. 
14 C. Zhao, Z. Ma, Q. Shao, B. Li, J. Ye and H. Peng, Energy Fuels, 2016, 30, 1154-1161.

15 J. Luo, L. Feng, Y. Chen, H. Sun, Q. Shen, X. Li and H. Chen, Water Res., 2015, 73, 332-341.

16 S. Kavitha, S. S. Pray, K. N. Yogalakshmi, S. A. Kumar and I. T. Yeom, Environ. Sci. Pollut. Res., 2016, 23(3), 2402-2414.

17 P. Eswari, S. Kavitha, S. Kaliappan, I. T. Yeom and J. R. Banu, Environ. Sci. Pollut. Res., 2016, 23(13), 13467-13479.

18 L. Appels, S. Houtmeyers, J. Degrève, J. Van Impe and R. Dewil, Bioresour. Technol., 2013, 128, 598-603.

19 J. H. Ahn, S. G. Shin and S. Hwang, Chem. Eng. J., 2009, 153(1), 145-150.

20 C. J. Chang, V. K. Tyagi and S. L. Lo, Bioresour. Technol., 2011, 102(17), 7633-7640.

21 S. Kavitha, J. R. Banu, J. V. Kumar and M. Rajkumar, Bioresour. Technol., 2016, 217, 21-27.

22 A. Tiehm, K. Nickel and U. Neis, Water Sci. Technol., 1997, 36(11), 121-128.

23 F. Passos, M. Solé, J. García and I. Ferrer, Appl. Energy, 2013, 108, 168-175.

24 APHA (American Public Health Association), Standard Methods for the Examination of Water and Wastewater, 20th edn, Washington, DC, USA, 1998.

25 D. Herbert, P. J. Philipps and R. E. Strange, Carbohydrate analysis, Methods Enzymol., 1971, 5B, 265-277.

26 O. H. Lowry, N. J. Rosebrough, A. L. Farr and R. J. Randall, J. Biol. Chem., 1951, 193(1), 265-275.

27 R. Goel, T. Mino, H. Satoh and T. Matsuo, Water Res., 1998, 32, 2081-2088.

28 H. Mu and Y. Chen, Water Res., 2011, 45(17), 5612-5620.

29 Y. Liu, X. Li, X. Kang, Y. Yuan, M. Jiao, J. Zhan and M. Du, Int. Biodeterior. Biodegrad., 2015, 102, 131-136.

30 X. Sun, X. Yuan, L. Chen, T. Wang, Z. Wang, G. Sun, X. Li, X. Li and G. Liu, Cell. Physiol. Biochem., 2017, 42(3), 11091119.
31 X. Du, Z. Shi, Z. Peng, C. Zhao, Y. Zhang, Z. Wang, X. Li, G. Liu and X. Li, J. Cell. Physiol., 2017, 232, 3296-3308.

32 Y. Song, N. Li, J. Gu, S. Fu, Z. Peng, C. Zhao, Y. Zhang, X. Li, Z. Wang, X. Li and G. Liu, J. Dairy Sci., 2016, 99(11), 91849198.

33 X. Zheng, Y. Su, X. Li, N. Xiao, D. Wang and Y. Chen, Environ. Sci. Technol., 2013, 47(9), 4262-4268.

34 C. Liu, H. Li, Y. Zhang, D. Si and Q. Chen, Bioresour. Technol., 2016, 216, 87-94.

35 J. Zhao, Q. Yang, X. Li, D. Wang, K. Luo, Y. Zhong, Q. Xu and G. Zeng, Waste Manag, 2015, 46, 133-139.

36 C. Zhao, X. Qiao, Y. Cao and Q. Shao, Fuel, 2017, 205, 184191.

37 C. Zhao, Q. Shao, Z. Ma, B. Li and X. Zhao, Ind. Crops Prod., 2016, 83, 86-93.

38 C. Huang, J. Lai, X. Sun, J. Li, J. Shen, W. Han and L. Wang, Bioresour. Technol., 2016, 220, 601-608.

39 X. Li, J. Zhao, D. Wang, Q. Yang, Q. Xu, Y. Deng, W. Yang and G. Zeng, Chemosphere, 2016, 144, 160-167.

40 M. T. Garcia, E. Campos, J. Sánchez-Leal and I. Ribosa, Water Res., 2006, 40(15), 2958-2964.

41 H. D. Ariesyady, T. Ito and S. Okabe, Water Res., 2007, 41(7), 1554-1568.

42 L. Feng, Y. Chen and X. Zheng, Environ. Sci. Technol., 2009, 43(12), 4373-4380.

43 D. Wang, Y. Liu, H. H. Ngo, C. Zhang, Q. Yang, L. Peng, D. He, G. Zeng, X. Li and B. J. Ni, Bioresour. Technol., 2017, 238, 343-351.

44 J. Zhao, D. Wang, X. Li, Q. Yang, H. Chen, Y. Zhong, H. An and G. Zeng, Sci. Rep., 2015, 5, 8602.

45 J. Zhao, Y. Liu, D. Wang, F. Chen, X. Li, G. Zeng and Q. Yang, Waste Manag., 2017, 67, 308-314. 